\title{
Dipolar interaction effects on the thermally activated magnetic relaxation of two-dimensional nanoparticle ensembles
}

\author{
S. I. Denisov a) and T. V. Lyutyy \\ Department of Mechanics and Mathematics, Sumy State University, 2, Rimskiy-Korsakov Street, \\ 40007 Sumy, Ukraine \\ K. N. Trohidou ${ }^{\text {b) }}$ \\ Institute of Materials Science, NCSR “Demokritos," 15310 Athens, Greece
}

(Received 21 October 2003; accepted 13 April 2004; published online 19 May 2004)

\begin{abstract}
The thermally activated magnetic relaxation in two-dimensional lattices of dipolar interacting nanoparticles with large uniaxial perpendicular anisotropy is studied by a numerical method and within the mean-field approximation for comparison. The role that the correlation effects play in magnetic relaxation and the influence of lattice structure and bias magnetic field on the relaxation process are revealed. The correlations of the nanoparticle magnetic moments enhance relaxation on small times, delay it on large times, and reduce the steady-state absolute magnetization at nonzero bias fields. In a hexagonal lattice, magnetic relaxation on small times occurs faster and the steady-state absolute magnetization has the larger magnitude than in a square lattice with the same lattice spacing. (C) 2004 American Institute of Physics. [DOI: 10.1063/1.1759782]
\end{abstract}

The two-dimensional (2D) ensembles of uniaxial ferromagnetic nanoparticles, whose easy axes of magnetization are perpendicular to the nanoparticles' plane, represent an important class of perpendicular magnetic recording media. ${ }^{1}$ From a technological point of view, the main characteristic of these media is the average time of data storage, which is one of the numerical parameters that describe the thermally activated magnetic relaxation. Due to the dipolar interaction between nanoparticles, its analytical description in such ensembles is a very complicated problem, which was solved only within the mean-field ${ }^{2,3}$ and fluctuating ${ }^{4}$ theories of magnetic relaxation. The former deals with the mean component of the dipolar field acting on the nanoparticles, and the latter accounts of both the mean and fluctuation components. However, the correlations of the nanoparticle magnetic moments, arising from the dipolar interaction, also play an important role, especially on the final phase of magnetic relaxation. To study the features of magnetic relaxation in such ensembles, which are conditioned by the correlation effects, we have developed a method for its numerical simulation at zero bias field. ${ }^{5}$ Usually, magnetic relaxation in ensembles of dipolar interacting nanoparticles is simulated either by the conventional Monte Carlo method, ${ }^{6,7}$ where the role of time plays the Monte Carlo steps, or by the improved Monte Carlo methods, ${ }^{8-11}$ that use the short-time stochastic behavior of the nanoparticle magnetic moments to convert the Monte Carlo steps to real time units. The main advantage of the method proposed in Ref. 5 is that it does not use the Monte Carlo approach, so the last complicated problem does not appear. This feature of the method and its applicability to the study of magnetic relaxation over ten decades of time ${ }^{5}$ make it a very useful tool in this domain.

In this letter, we extend our method to the case of non-

\footnotetext{
${ }^{a)}$ Electronic mail: denisov@sumdu.edu.ua

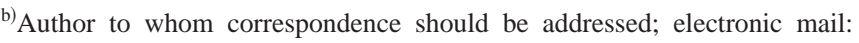
trohidou@ims.demokritos.gr
}

zero bias magnetic fields, and we present results obtained within its framework, which clarify the role that the correlation effects, bias field, and lattice structure play in the magnetic relaxation. We consider the $2 \mathrm{D}$ ensembles of spherical nanoparticles with a radius $r$, which occupy the sites of a square or hexagonal lattice with lattice spacing $d$, and whose easy axes of magnetization are perpendicular to the lattice plane ( $x y$ plane). We also assume that: (a) the nanoparticle magnetic moments $\mathbf{m}_{i}(t)$ (the index $i$ labels the nanoparticles) perform a coherent rotation $\left(\left|\mathbf{m}_{i}(t)\right|=m\right)$, (b) a bias magnetic field $H_{0} \mathbf{k}$ (k is the unit vector along the $z$ axis) acts on each $\mathbf{m}_{i}(t)$, and (c) the initial condition $\mathbf{m}_{i}(0)=m \mathbf{k}$ holds for all $i$.

If the potential barriers between the equilibrium directions of all $\mathbf{m}_{i}(t)$ are much larger than the thermal energy $k_{B} T$ ( $k_{B}$ is the Boltzmann constant, $T$ is the absolute temperature), then the vectors $\mathbf{m}_{i}(t)$ fluctuate within small vicinities of the $\mathbf{k}$ and $\mathbf{- k}$ directions, and they are reoriented occasionally. In this case the average numbers $N_{+}(t)$ and $N_{-}(t)$ of positively and negatively oriented magnetic moments in a lattice region that contains $N(\gg 1)$ nanoparticles are well defined $\left(N_{+}(t)+N_{-}(t) \approx N\right)$, and we can introduce the reduced magnetization of the nanoparticle ensemble as $\rho(t)=2 N_{+}(t) / N-1$. To calculate the relaxation law $\rho(t)$ at $H_{0}=0$, we have developed the procedure ${ }^{5}$ that is based on the analytical determination of the probability reorientation of the nanoparticle magnetic moments, and on the numerical determination of the nanoparticle ensemble states. Its important feature consists of the fact that the real dipolar fields acting on $\mathbf{m}_{i}(t)$ are taken into account.

Here, we generalize this procedure to the $H_{0} \neq 0$ case and apply it to study the magnetic relaxation in ensembles of $\mathrm{Co}$ nanoparticles. The generalization consists of the derivation of the exact formula for the calculation of the probability reorientation of the nanoparticle magnetic moments for $H_{0} \neq 0$. We use this formula, derived from the corresponding backward Fokker-Planck equation, and the numerical proce- 


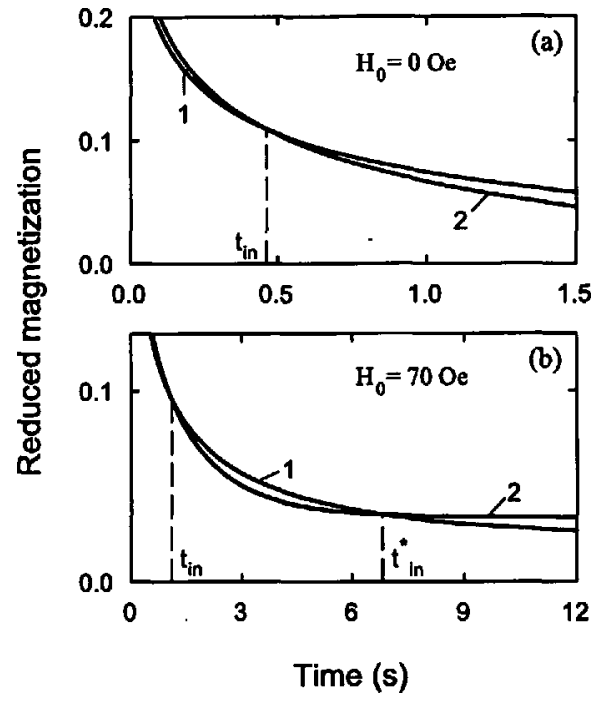

FIG. 1. Plots of $\rho^{s}(t)$ (curve 1) and $\rho_{\mathrm{mf}}^{s}(t)$ (curve 2) for $d^{s}=3 r$.

dure of Ref. 5 to calculate the relaxation law $\rho(t)$ in these ensembles. We choose the basic nanoparticle ensemble for our simulation in the form of square (for square lattice) or hexagonal (for hexagonal lattice) box and surround it by eight or six identical ensembles, respectively. To calculate the dipolar field acting on a nanoparticle, we consider this nanoparticle as the central one in the box of the same size as the basic and we include the nanoparticles, which belong to this box. Increase of the size of the basic box leads to reduction of the boundary effects and, to practically eliminate them, we chose the size of the square and hexagonal boxes equal to $100 d$ and $60 d$, respectively.

To gain more insight into the magnetic relaxation, we also calculate the relaxation law $\rho_{\mathrm{mf}}(t)$ within the mean-field approximation. As this approach ignores the correlation effects, the difference between $\rho(t)$ and $\rho_{\mathrm{mf}}(t)$ results from the correlations of the nanoparticle magnetic moments. In what follows, the superscripts ${ }^{s}$ and ${ }^{h}$ on $\rho(t), \rho_{\mathrm{mf}}(t)$, and $d$ denote the square and hexagonal lattice, respectively.

The role that the correlation effects and the bias field $H_{0}$ play in magnetic relaxation is illustrated in Fig. 1. We present the numerical results for ensembles of Co nanoparticles characterized by the parameters $H_{a}=6400 \mathrm{Oe}\left(H_{a}\right.$ is the anisotropy field), $m / V=1400 \mathrm{G}$ ( $V$ is the nanoparticle volume), $\lambda=0.2$ ( $\lambda$ is the Landau-Lifshitz damping parameter), $r=4 \mathrm{~nm}$, and $T=300 \mathrm{~K}$. Due to the correlations of the nanoparticle magnetic moments, for $H_{0}=0$ the actual magnetic relaxation occurs faster on small times and more slowly on large times, than the mean-field theory predicts [see Fig. 1(a)]. In this case the curves $\rho^{s}(t)$ and $\rho_{\mathrm{mf}}^{s}(t)$ are intersected at the time $t=t_{\text {in }}$, and $\rho^{s}(\infty)=\rho_{\mathrm{mf}}^{s}(\infty)=0$. In equilibrium, the local dipolar fields have, on average, the same directions as the magnetic moments and the mean dipolar field equals zero (for $H_{0}=0$ ), therefore for $H_{0} \neq 0$ the condition $\left|\rho^{s}(\infty)\right|$ $<\left|\rho_{\mathrm{mf}}^{s}(\infty)\right|$ must hold. For $H_{0}<0$ we have $\rho_{\mathrm{mf}}^{s}(\infty)<\rho^{s}(\infty)<0$, and the curves $\rho^{s}(t)$ and $\rho_{\mathrm{mf}}^{s}(t)$ are intersected only once, as in the $H_{0}=0$ case. If $H_{0}>0$, then $\rho_{\mathrm{mf}}^{s}(\infty)>\rho^{s}(\infty)>0$, for small enough values of $H_{0}$ these curves are intersected twice [see Fig. 1(b)], with increasing $H_{0}$ the time interval $\left(t_{\text {in }}, t_{\text {in }}^{*}\right)$ is decreasing, and $\rho_{\mathrm{mf}}^{s}(t)$ $>\rho^{s}(t)(t>0)$ for large $H_{0}$ [see Fig. 2(a), curves 1 and 2]. Downloaded 28 May 2004 to 140.105 .16 .2 . Redistribution subje.

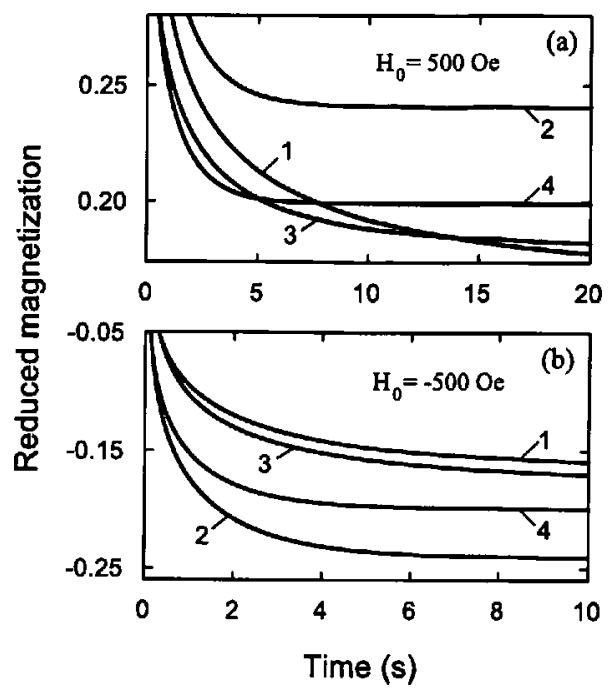

FIG. 2. Plots of $\rho^{s}(t)$ (curve 1), $\rho_{\mathrm{mf}}^{s}(t)$ (curve 2), $\rho^{h}(t)$ (curve 3), and $\rho_{\mathrm{mf}}^{h}(t)$ (curve 4) for $d^{s}=d^{h}=3 r$.

The features of magnetic relaxation in ensembles of Co nanoparticles, which arise from the different nanoparticle arrangement in square and hexagonal lattices, are demonstrated in Figs. 2 and 3. If the lattice spacing $d$ is the same for both lattices (Fig. 2), then the difference between the relaxation laws results mainly from the different number of the nearest sites in these lattices ( 4 vs 6 ). At $t=0$ the local dipolar fields in hexagonal lattices are larger than in square ones, so the initial phase of magnetic relaxation occurs faster in hexagonal lattices. At $t=\infty$ and $H_{0}=0$ the conditions $\rho^{s}(\infty)=\rho^{h}(\infty)=0$ and $\rho_{\mathrm{mf}}^{s}(\infty)=\rho_{\mathrm{mf}}^{h}(\infty)=0$ hold. Each magnetic moment in a square lattice is surrounded, on average, by four opposite oriented magnetic moments, whereas in a hexagonal lattice - by four opposite and by two similarly oriented magnetic moments. Therefore, if $H_{0}>0$ [see Fig. 2(a)] then $\rho_{\mathrm{mf}}^{s}(\infty)>\rho_{\mathrm{mf}}^{h}(\infty)>0, \rho^{h}(\infty)>\rho^{s}(\infty)>0$, and the curves $\rho^{s}(t)$ and $\rho^{h}(t)$ have the unique intersection point $t$ $=t_{1}\left(t_{1} \approx 13.59 \mathrm{~s}\right.$ for $\left.H_{0}=500 \mathrm{Oe}\right)$. If $H_{0}<0$ [see Fig. 2(b)] then $\rho_{\text {mf }}^{s}(\infty)<\rho_{\text {mf }}^{h}(\infty)<0$ and, since $\rho^{h}(\infty)<\rho^{s}(\infty)<0$, the

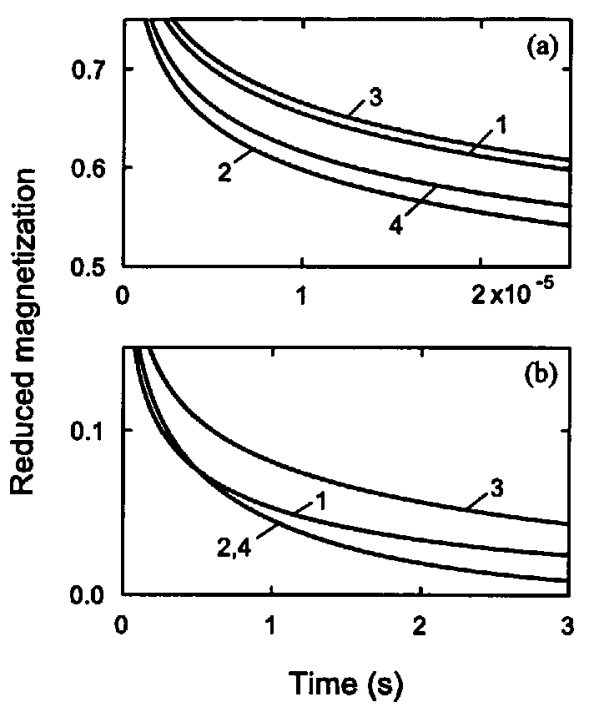

FIG. 3. Plots of $\rho^{s}(t)$ (curves 1), $\rho_{\mathrm{mf}}^{s}(t)$ (curves 2), $\rho^{h}(t)$ (curves 3), and $\rho_{\mathrm{mf}}^{h}(t)$ (curves 4) on small (a) and large (b) time scales for $d^{h}=3 r, d^{s}$ $=d^{h} 3^{1 / 4} / 2^{1 / 2}$ and $H_{0}=0$. 
curves $\rho^{s}(t)$ and $\rho^{h}(t)$ have two intersection points $t=t_{1}$ and $t=t_{2}\left(t_{1} \approx 0.01 \mathrm{~s}, t_{2} \approx 0.33 \mathrm{~s}\right.$ for $\left.H_{0}=-500 \mathrm{Oe}\right)$.

Figure 3 presents the character of magnetic relaxation in square and hexagonal lattices, with unit cells that have the same area, i.e., $\left(d^{s}\right)^{2}=\left(d^{h}\right)^{2} \sqrt{3} / 2$, at $H_{0}=0$. Despite the fact that each site in the hexagonal lattice has more nearest neighbors than the square one, the local and mean dipolar fields in the square lattice exceed the corresponding fields in the hexagonal one, since $d^{s}<d^{h}\left(d^{s} \approx 0.931 d^{h}\right)$. Therefore the conditions $\rho^{h}(t)>\rho^{s}(t)$ and $\rho_{\mathrm{mf}}^{h}(t)>\rho_{\mathrm{mf}}^{s}(t)$ must hold for all $t>0$ [at large times (see Fig. 3(b)) the distinction between $\rho_{\mathrm{mf}}^{h}(t)$ and $\rho_{\mathrm{mf}}^{s}(t)$ is not visible on the chosen scale of time]. On the other hand, for the same reasons as in the case presented in Fig. 1(a), the curves $\rho^{s}(t)$ and $\rho_{\mathrm{mf}}^{s}(t)$, and $\rho^{h}(t)$ and $\rho_{\mathrm{mf}}^{h}(t)$ are intersected once at $t=t_{\text {in }}\left(t_{\text {in }} \approx 0.505 \mathrm{~s}\right.$ and $6.597 \times 10^{-3} \mathrm{~s}$ for the square and hexagonal lattice, respectively).

Sometimes the coefficient of magnetic viscosity $S=-d \rho(t) / d \ln t$ is used, to characterize the magnetic properties of nanoparticle ensembles. If $S \approx$ constant on a wide time interval, as in the case of log-normal distribution for nanoparticle diameters, then the parameter $S$ is an important characteristic of the nanoparticle ensemble, which defines the relaxation law on that interval. For the considered ensembles, $S$ depends strongly on $t$, therefore this parameter does not play a special role in our case.

In conclusion, we have generalized the method developed in Ref. 5 to study the magnetic relaxation in 2D ensembles of dipolar interacting nanoparticles subjected to a bias magnetic field. We have demonstrated its efficiency for the analysis of the relaxation law features arising from the mutual action of the dipolar correlations and bias field for different ensembles of Co nanoparticles and we have shown that the correlation effects play an important role in magnetic relaxation on all times. We expect that, because of the abovementioned unique resources of the proposed method, it can be useful for the design of more reliable perpendicular nanoparticle media.

${ }^{1}$ For a review see, for example, The Physics of Ultra-High-Density Magnetic Recording, edited by M. L. Plumer, J. Van Ek, and D. Weller (Springer, Berlin, 2001); A. Moser, K. Takano, D. T. Margulies, M. Albrecht, Y. Sonobe, Y. Ikeda, S. Sun, and E. E. Fullerton, J. Phys. D 35, R157 (2002).

${ }^{2}$ D. K. Lottis, R. M. White, and E. D. Dahlberg, Phys. Rev. Lett. 67, 362 (1991).

${ }^{3}$ S. I. Denisov and K. N. Trohidou, Phys. Status Solidi A 189, 265 (2002).

${ }^{4}$ S. I. Denisov and K. N. Trohidou, Phys. Rev. B 64, 184433 (2001).

${ }^{5}$ S. I. Denisov, T. V. Lyutyy, and K. N. Trohidou, Phys. Rev. B 67, 014411 (2003).

${ }^{6}$ L. C. Sampaio, R. Hyndman, F. S. de Menezes, J. P. Jamet, P. Meyer, J. Gierak, C. Chappert, V. Mathet, and J. Ferré, Phys. Rev. B 64, 184440 (2001).

${ }^{7}$ M. Ulrich, J. García-Otero, J. Rivas, and A. Bunde, Phys. Rev. B 67, 024416 (2003).

${ }^{8}$ Y. Kanai and S. H. Charap, IEEE Trans. Magn. 27, 4972 (1991).

${ }^{9}$ R. W. Chantrell, N. Walmsley, J. Gore, and M. Maylin, Phys. Rev. B 63, 024410 (2000).

${ }^{10} \mathrm{U}$. Nowak, in Annual Reviews of Computational Physics IX, edited by D. Stauffer (World Scientific, Singapore, 2001), p. 105.

${ }^{11}$ R. J. M. van de Veerdonk, X. W. Wu, R. W. Chantrell, and J. J. Miles, IEEE Trans. Magn. 39, 590 (2003). 